
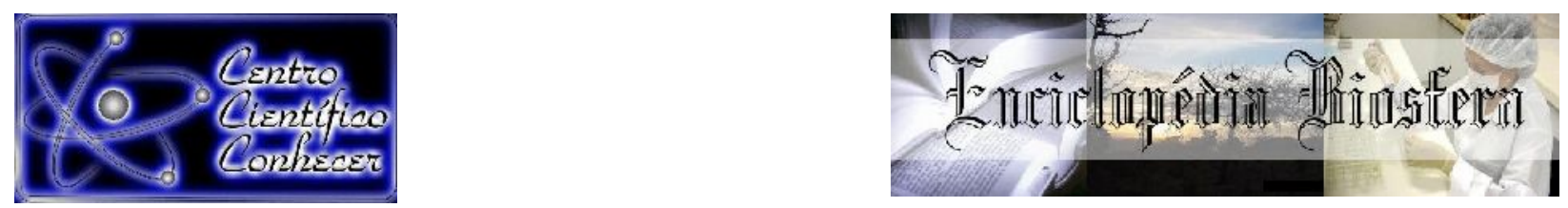

\title{
TEMPOS DE CONTATO E NÍVEIS DE MISTURA DE UREIA COM SEMENTES DE BRAQUIÁRIA BRIZANTHA CV. MARANDU PARA SISTEMAS INTEGRADOS
}

Danilo Cordeiro Maciel ${ }^{1}$, Roberta Santos Santiago Amaral ${ }^{2}$, Laís Lopes Pereira ${ }^{1}$, Dea Alécia Martins Netto $^{3}$, Alexandre Martins Abdão dos Passos ${ }^{3}$

${ }^{1}$ Estudante do Curso de Agronomia da Universidade Federal de São João del-Rei, Sete Lagoas, Minas Gerais, Brasil. Bolsistas PIBIC CNPq/Embrapa.

2 Estudante do curso de Ciências Biológicas da UNIFEMM, Sete Lagoas, Minas Gerais, Brasil. bolsista Embrapa

${ }^{3}$ Pesquisadores da Embrapa Milho e Sorgo, Sete Lagoas, Minas Gerais, Brasil. Email: alexandre.abdao@embrapa.br

Recebido em: 04/10/2019 - Aprovado em: 30/11/2019 - Publicado em: 15/12/2019 DOI: 10.18677/EnciBio_2019B55

A utilização de sementes de alta qualidade é uma premissa para a formação de pastos visando à geração de sistemas de produção sustentáveis na produção pecuária. Uma prática comum é a aplicação à lanço de sementes nuas de braquiária em mistura com fertilizantes nitrogenados ou fosfatados. Objetivou-se estudar o efeito de seis tempos de contato $(3,12,24,48$ e 72 horas) de ureia granulada com sementes de alta qualidade (alto valor cultural) de Brachiaria brizantha cultivar Marandu, na sua qualidade fisiológica. O delineamento experimental utilizado foi o inteiramente casualizado com tratamento adicional (sementes sem fertilizante) com quatro repetições. Foram avaliadas a porcentagem de emergência em areia, porcentual de germinação de plântulas normais, anormais e sementes mortas sobre papel em caixas gerbox em condições controladas de fotoperíodo e temperatura (35 ${ }^{\circ} \mathrm{C}$ por 8 horas de radiação e $20^{\circ} \mathrm{C}$ por 16 horas no escuro). Verificou-se que com 72 horas de contato, todas as sementes apresentaram perda de viabilidade. $O$ número de sementes mortas apresentou correlação significativa $(p<0,001)$ e negativa de $98,63 \%$ para o número de plântulas normais. A proporção de mistura entre ureia e semente influencia a velocidade de deterioração das sementes, expressa em menor porcentagem de plântulas emergidas em areia em relação às sementes que não entraram em contato com a ureia (testemunha). Conclui-se que as iniciam as perdas de viabilidade e vigor a partir de 3 horas de contato com 0 fertilizante ureia, tendo um pronunciado aumento no número de sementes inviáveis a partir de 48 horas.

PALAVRAS-CHAVE: Deterioração; Tecnología de sementes; Urochloa brizantha 


\title{
BRAQUIARIA BRIZANTHA CV. MARANDU UNDER CONTACT TIMES AND UREA MIXING LEVELS TO INTEGRATED PASTURE SYSTEMS
}

\begin{abstract}
The use of high-quality seeds is a premise for the implantation of pasture for the generation of sustainable farming systems in livestock production. A usual practice is to mix brachiaria seeds with nitrogenous or phosphate fertilizers to sown and sidedressing. The objective of this study was to study the effect of contact time $(0,3$, 12, 24, 48 and 72 hours) of urea mineral fertilizer with Brachiaria brizantha cv. Marandu high-quality seeds, in its physiological quality (germination and vigor). A completely randomized experimental design with additional treatment (seeds without fertilizer) was used, with three replicates, in laboratory and chamber conditions. Percentage of seedling emergence, germination of normal and abnormal seedlings and dead seeds were evaluated. Seeds were sown over the papers placed in gerboxes under controlled conditions of photoperiod and temperature $\left(35^{\circ} \mathrm{C}\right.$ for 8 hours of light and $20^{\circ} \mathrm{C}$ for 16 in dark conditions). It was found that after 72 hours of contact, all seeds lose its viability. The number of dead seeds presented significant $(p<0.001)$ and negative correlation of $98.63 \%$ for the number of normal seedlings. The mix ratio between urea and seed affects the rate of seed deterioration, expressed as the lowest percentage of seedlings emerged in the sand, in relation to seeds not in contact with urea (control). It is concluded that from the reduction in viability and vigor begin after 3 hours of contact with fertilizers, so the number of unviable seeds highly increase after 48-hours contact.
\end{abstract}

KEYWORDS: Deterioration; Seed technology; Urochloa brizantha

\section{INTRODUÇÃO}

A pastagem é um dos principais usos do solo no território brasileiro, sendo grande parte cultivada com gramíneas do gênero Braquiária. Dessas áreas, significativa parte está em avançado processo de degradação agronômica e biológica (TORRES et al., 2019; VALLE JUNIOR et al., 2019), havendo muitas áreas implantadas em solos de baixa fertilidade, ou apresentando declínio devido a utilização de práticas inadequadadas de manejo.

Sabe-se que os sistemas integrados com lavoura e pecuária representam uma alternativa de produção racional que promove a recuperação das pastagens gerando diversos benefícios à atividade pecuária (CARVALHO et al., 2018). Especialmente para solos frágeis, tais como os arenosos, localizados em áreas de alta variabilidade climática, dentre outros os sistemas integrados aumenta o aporte de palhada, gerando matéria orgânica que possui alta capacidade de retenção de água e nutrientes, dando condições de desenvolvimento das plantas forrageiras em novas áreas agrícolas, otimizando o uso da água e do solo (LOPES, GUILHERME 2016; MORAES et al., 2016; PHILP et al., 2019).

Visando à implantação dos sistemas integrados uma técnica utilizada é o consórcio de plantas anuais, com gramíneas, frequentemente no cerrado brasileiro, utilizando o milho, sorgo e soja com braquiária (CAGNA et al., 2019). Em sistemas de consórcio simultâneo, uma alternativa de implantação, é a semeadura conjunta da braquiária e aplicação de fertilizantes, podendo ser durante a implantação da cultura granífera ou em sobressemeadura durante a adubação de cobertura (MATEUS et al., 2016; ALMEIDA et al., 2017). 
A adubação nitrogenada em cobertura é uma prática agronômica consolidada para diversas espécies cultivadas (SPACKMAN et al., 2019), fornecendo o nutriente no tempo mais apropriado, de maior demanda da cultura estabelecida (PANISON et al., 2019). A semeadura da braquiária nesse momento, após o estabelecimento da cultura principal, visa estabelecer uma pastagem com alto valor bromatológico e de alto rendimento de biomassa (TAFFAREL et al., 2018), com uma menor interferência na produtividade da cultura principal. Pode-se ainda citar como benefício a redução no uso de tempo e combustível, evitando a aplicação isolada de cada insumo, uma vez que vários produtores não possuem máquinas e implementos adaptados para a semeadura simultânea de pequenas sementes .

A utilização de sementes com atributo de qualidade fisiológica é um dos requisitos para a formação de uma lavoura bem estabelecida (JOHANN et al., 2019). Nesse contexto, a mistura de sementes com fertilizantes representa um fator de estresse e incremento da taxa de deterioração das sementes. Fertilizantes minerais apresentam diversas características que promovem decréscimo na qualidade fisiológica das sementes (CAVARIANI et al., 1994; MATEUS et al., 2007; DAN et al., 2011; CODOGNOTO et al., 2019) ocasionando perdas de viabilidade e vigor (LIMA et al., 2010; PERES et al., 2012). Dessa forma, a busca por alternativas, tais como a utilização de sementes de alto vigor, recobrimento especiais das sementes e o máximo tempo de contato é primordial para a viabilização e otimização do uso dos fatores de produção em sistemas integrados. O conhecimento da perda de vigor e viabilidade das sementes é primordial para o melhor planejamento do agricultor para utilização ou não da mistura prévia de sementes de braquiárias e fertilizantes.

Objetivou-se avaliar períodos de contato de sementes de Brachiaria brizantha cultivar Marandu, de alta qualidade fisiológica e física, com doses crescentes de ureia visando a semeadura simultânea para formação de pastagem.

\section{MATERIAL E MÉTODOS}

O trabalho foi realizado no Laboratório de Análise de semente da Embrapa Milho e Sorgo em Sete Lagoas, Minas Gerais. Avaliou-se o efeito de cinco tempos de contato $(3,12,24,48$ e 72 horas) de sementes da braquiária brizantha cultivar Marandu com três diferentes doses de ureia $(50,100$ e $150 \mathrm{~kg}$ de nitrogênio por hectare) utilizando-se delineamento estatístico inteiramente casualizado, e avaliando a viabilidade e vigor das plântulas. Como teste controle (testemunha) foi montado um tratamento adicional, no qual as sementes não tiveram contato com o fertilizante.

Para a definição das proporções utilizou-se uma taxa de semeadura de 520 pontos de valor cultura por hectare para a Braquiária brizantha cv. Marandu, com sementes nuas e sem tratamento, apresentando $90 \%$ de valor cultural. A mistura foi feita em embalagens plásticas devidamente homogeneizadas e mantidas em temperatura controlada laboratorial de $25^{\circ} \mathrm{C}$. Decorrido o tempo de contato avaliado, a separação das sementes do fertilizante foi realizada por via úmida, utilizando-se água deionizada e agitador magnético, sendo as sementes posteriormente retiradas da água por meio de peneira e secas sobre papel toalha e semeadas.

As sementes foram dispostas em caixas gerbox sobre duas folhas de papel em germinadores com fotoperíodo e temperaturas controladas específicas para a espécie avaliada $\left(35^{\circ} \mathrm{C}\right.$ por 8 horas de radiação e $20^{\circ} \mathrm{C}$ por 16 horas na ausência de luz), adicionado uma massa de água compreendendo 2,5 vezes a do papel.

Para o teste de emergência foram utilizadas bandejas contendo neossolo quartzarênico. As bandejas foram acondicionadas em uma câmara de crescimento 
simulando as temperaturas noturnas de $20^{\circ} \mathrm{C}$ e diurna de $35^{\circ} \mathrm{C}$, apropriadas para as braquiárias. Foi realizada a determinação da capacidade de campo do solo $(0,17$ $\mathrm{dm}^{3} \mathrm{dm}^{-3}$ ) e retenção de água previamente ao experimento para determinar o volume de água a ser aplicado na semeadura (60\% da capacidade de campo) e a manutenção até a avaliação. A parcela experimental foi constituída de 50 sementes puras viáveis prévia e manualmente separadas.

As proporções do fertilizante nitrogenado e sementes utilizadas tomaram como referência a aplicação de três níveis de fertilizantes, recomendadas para diferentes regiões e condições de cultivo de milho, compreendendo (50, 100 e 150 $\mathrm{kg}$ de $\mathrm{N} \mathrm{ha}^{-1}$ ), e contendo $46 \%$ de $\mathrm{N}$, apresentando um índice de salinidade de $76 \%$ em relação ao nitrato de sódio.

Foram realizadas leituras ao final de 21 dias determinando-se o número de plântulas emergidas e sementes mortas, plântulas anormais e normais no ensaio de germinação (BRASIL, 2009). Os valores computados foram posteriormente transformados em porcentagem. Foram consideradas plântulas anormais aquelas que não apresentavam todas as estruturas primárias, desproporções dentre essas e comprimento da parte aérea menor que $20 \mathrm{~mm}$. Plântulas normais aquelas com radícula e parte aérea presentes e plenamente desenvolvidas. Verificou-se a emergência em canteiro contabilizando-se as plântulas emergidas.

Para a realização da análise de variância foram verificados os pressupostos pelos testes de Shapiro-Wilk e Levene. Foi aplicada a análise de variância, seguida pelo teste de Scott knott e realização de regressões para ajustes aos dados temporais. As análises estatísticas foram realizadas com auxílio do software estatístico AgroEstat ${ }^{\circledR}$ (BARBOSA; MALDONADO JÚNIOR, 2010).

\section{RESULTADOS E DISCUSSÃO}

Verificou-se efeitos dos tempos de exposição sobre a emergência das plântulas em areia, sobre o porcentual de germinação de plântulas normais, anormais e sementes mortas de braquiária brizantha Marandu (Tabela 1). Por sua vez, as plântulas emergidas foram diferentemente afetadas pelo tempo de exposição de acordo com os níveis de ureia utilizados. O tratamento adicional, sem contato com a ureia, diferiu da média dos tratamentos com ureia em todas as variáveis analisadas. A proporção de ureia e sementes não afetou a viabilidade das sementes, nem o vigor das plântulas.

TABELA 1 - Resumo da análise de variância para emergência em areia, porcentual de germinação de plântulas normais, anormais e sementes mortas de braquiária Marandu sob diferentes tempos de contato e doses de ureia para semeadura simultânea à adubação

\begin{tabular}{lccccc}
\hline Causas de Variação & GL & Emergência & Normais & Anormais & Mortas \\
\hline Nitrogênio (N) & 2 & 0,1092 & 0,6636 & 0,3392 & 0,3386 \\
Tempo (T) & 4 & $<0,0001$ & $<0,0001$ & 0,0022 & $<0,0001$ \\
$\mathbf{N}$ x T & 8 & 0,0455 & 0,2833 & 0,7528 & 0,1617 \\
\hline (Fatorial) & 14 & $<0,0001$ & $<0,0001$ & 0,0495 & $<0,0001$ \\
Adicional vs. Fatorial & 1 & $<0,0001$ & $<0,0001$ & 0,2645 & $<0,0001$ \\
\hline (Tratamentos) & 15 & $<0,0001$ & $<0,0001$ & 0,0522 & $<0,0001$ \\
Resíduo & 48 & & & & \\
\hline
\end{tabular}

\section{Total}

Significância a 5 e 1\% pelo teste $\mathrm{F}$, respectivamente. 
A exposição das sementes de braquiária com adubos, potássicos, nitrogenados e fosfatados promove um incremento na deterioração da qualidade fisiológica das mesmas (MATEUS et al., 2007). Diversos autores reportam que os níveis de degradação são correlacionados com o tempo de exposição (LIMA et al., 2010; LIMA et al., 2014), uma vez que os efeitos deletérios dos fatores de estresse são aditivos (BEWLEY et al., 2013).

A emergência das plântulas oriundas das sementes expostas ao fertilizante apresentou comportamento diferenciado ao longo do tempo de contato para cada proporção de adubo utilizada (Tabela 2). No geral, verificou-se perdas significativas de vigor a partir de 24, 12 e 3 horas respectivamente para os níveis de 50, 100 e 150 $\mathrm{kg}$ de nitrogênio por hectare, demonstrando que os maiores níveis, aceleram a deterioração das sementes de braquiária brizantha Marandu. Já foi verificado que sementes de Urochloa brizantha cultivar Piatã sofreram perda de vigor proporcional ao tempo de exposição à sais de nitrato de cálcio $\left[\mathrm{Ca}\left(\mathrm{NO}_{3}\right)^{2}\right]$ e sulfato de cálcio $\left(\mathrm{CaSO}_{4}\right)$ demonstrando diferença de apenas 12 horas incrementos na condutividade elétrica e emergência em areia (RIBEIRO et al., 2019). O potencial osmótico é dependente da concentração do soluto, portanto, quanto maior for a relação de ureia, fertilizante nitrogenado que apresenta elevado poder de salinidade relativa, maior será o efeito de estresse de dessecação sobre os tecidos externos e membranas nas sementes (BEWLEY et al., 2013; RIBEIRO et al., 2019).

TABELA 2 - Médias de emergência em areia para braquiária brizantha Marandu e níveis de ureia em mistura sementes: ureia

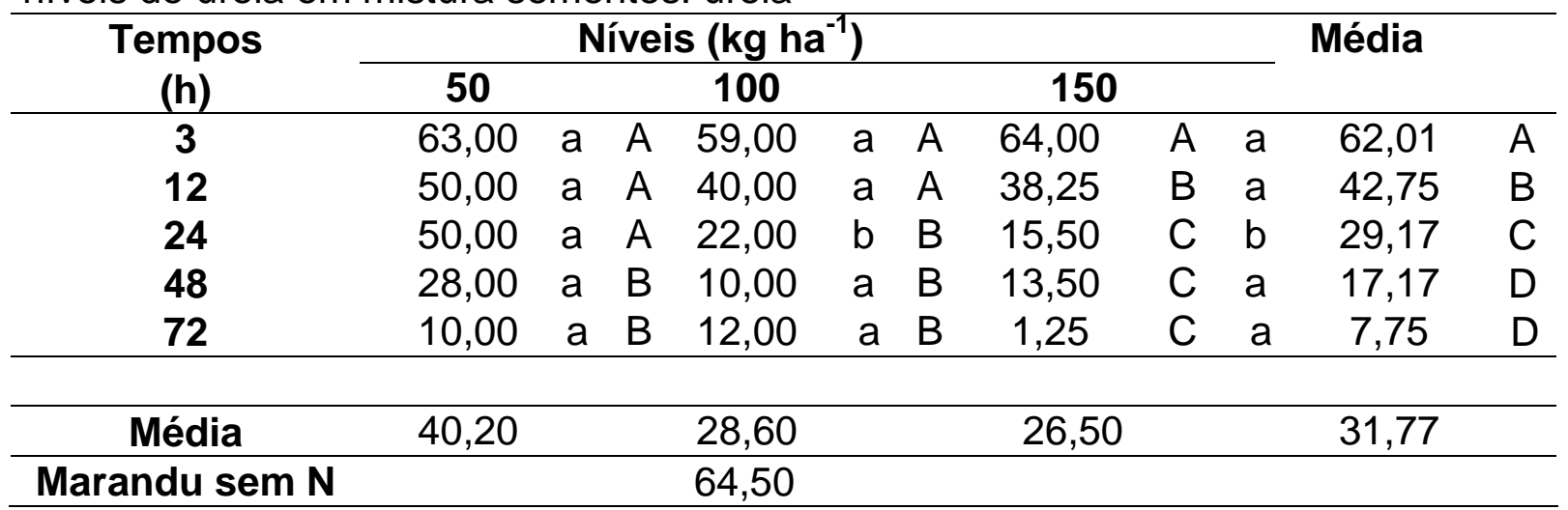

Médias seguidas da mesma letra, maiúscula na linha e minúscula na coluna, não diferem entre si, pelo teste de Scott Knott $(p<0.05)$.

Não foi verificado efeito dentro de cada tempo de exposição para os diferentes níveis de ureia utilizados nas misturas de fertilizante com as sementes, exceto no tempo de 24 horas, no qual, a menor proporção, utilizando-se $50 \mathrm{~kg}$ de N por hectare em forma de ureia, diferiu dos demais níveis (Tabela 2). As sementes que não foram misturadas ao fertilizante, apresentaram um elevado potencial fisiológico, com uma emergência média de $64,5 \%$, considerada como um lote de alto vigor e qualidade para os padrões nacionais (SILVA et al., 2018). Apesar da média dos tratamentos que tiveram contato com o fertilizante diferirem da média do lote sem mistura, observa-se que os tratamentos com apenas três horas de contato permaneceram com um elevado vigor, tendo a partir desse tempo, perdas significativas. 
Por sua vez, o número de sementes mortas, isto é, sem viabilidade, aumentaram significativamente a partir das 48 horas de exposição ao fertilizante, independente na razão de mistura entre adubo e sementes (Figura 1). Similarmente, a razão de plântulas normais, descreveu significativamente a partir dos dois dias de exposição, alcançando valores próximos de zero às 72 horas de exposição.

No processo de deterioração das sementes, foi verificada uma sequência de reações em nível intracelular até degradação de tecidos. Inicialmente as sementes perdem 0 vigor, podendo ainda permanecerem viáveis, mantendo o poder germinativo (BEWLEY et al., 2013). Contudo, mesmo no desenvolvimento, após a germinação, as plântulas expressam a perda de vigor, apresentando baixa resiliência e resistência às condições adversas no solo, o que pode expressar um estabelecimento inicial de lavouras abaixo do esperado, com reflexos negativos sobre a produtividade primária das plantas.

Com o avanço do processo de deterioração, diversas estruturas e organelas são dizimadas, o que promove em último caso, a morte do embrião e a perda de viabilidade da semente (BEWLEY et al., 2013). Diferentes lotes de sementes podem apresentar, portanto, a mesma viabilidade, expressa geral e diretamente em poder germinativo ou, no caso de sementes de braquiárias, indiretamente por testes de tetrazólico (SILVA et al., 2018), mas apresentarem vigores diferenciados, pois se encontram em etapas distintas na cadeia de deterioração.

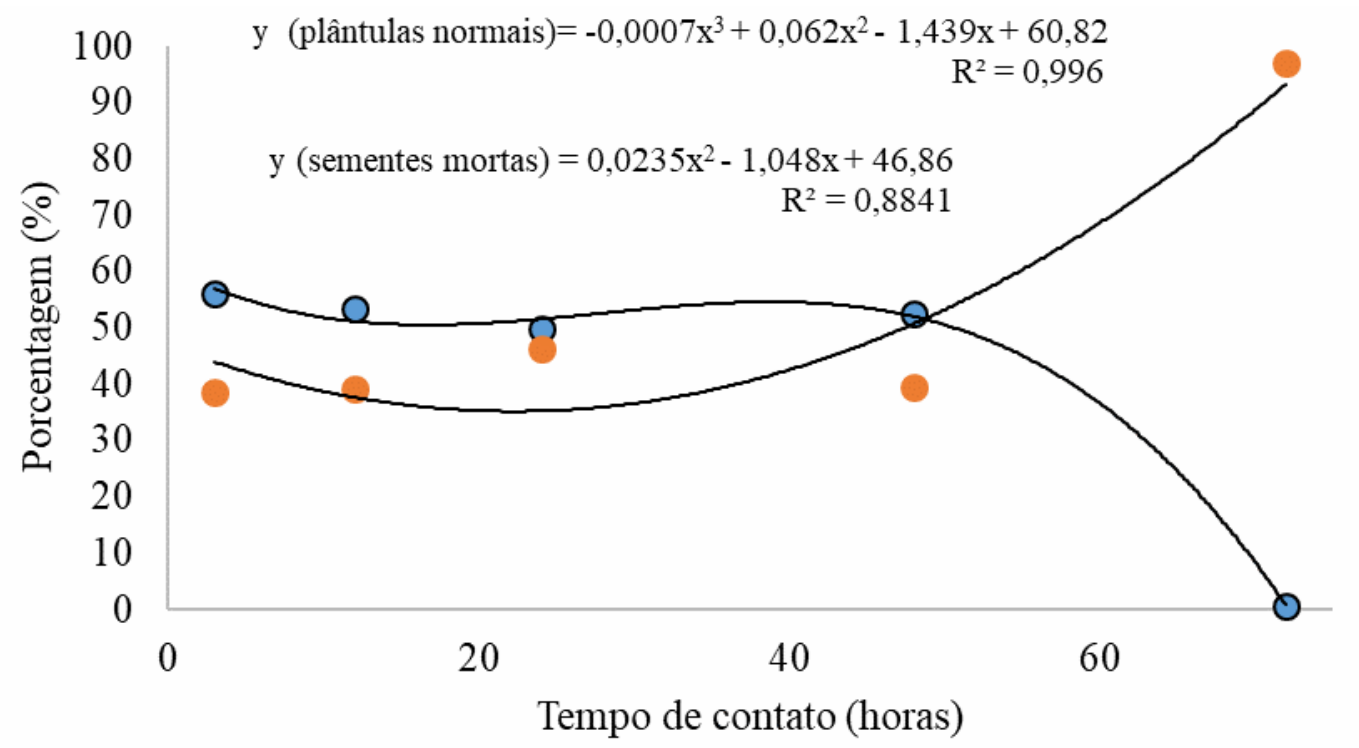

FIGURA 1. Porcentagem de sementes mortas e plântulas normais em função do tempo de exposição das sementes com o fertilizante ureia.

Dentre as variáveis respostas, observou-se uma significativa e alta correlação negativa entre as proporções de sementes mortas e sementes viáveis formadoras de plântulas normais (Tabela 3). Apesar de se verificar correlação significativa entre as plântulas normais e emergência em canteiro de areia, plântulas anormais e sementes mortas e essas com a emergência em canteiro, as correlações foram baixas (Tabela 3 ). 
TABELA 3 - Correlação simples para os ensaios de viabilidade de sementes e vigor de plântulas

\begin{tabular}{lccc}
\hline & Normais & Anormais & Mortas \\
\hline Anormais & $0,16461^{\mathrm{NS}}$ & - & - \\
& 0,1937 & - & - \\
Mortas & $-0,9863^{* *}$ & $-0,3248^{\star *}$ & - \\
& $<0,0001$ & 0,0088 & - \\
\multirow{4}{*}{ Emergência } & & & \\
& $0,61086^{* *}$ & $0,05174^{\mathrm{NS}}$ & $-0,5944^{* *}$ \\
& $<0,0001$ & 0,6847 & $<0,0001$ \\
\hline
\end{tabular}

\section{CONCLUSÕES}

Sementes de Braquiária brizantha cultivar Marandu de alta qualidade fisiológica iniciam as perdas de viabilidade e vigor a partir de três horas de contato com o fertilizante ureia.

Quanto maior a proporção de mistura entre ureia e sementes, maior é a velocidade de deterioração das sementes, expressa em menor porcentagem de plântulas emergidas em areia.

\section{REFERÊNCIAS}

ALMEIDA, R.M.E, GOMES, CAIO M.; LAGO, B.C; OLIVEIRA, S.M. et al. Corn yield, forage production and quality affected by methods of intercropping corn and Panicum maximum. Pesquisa Agropecuária Brasileira, 52(3), 170-176.2017. Disponível em <https://dx.doi.org/10.1590/s0100-204×2017000300004> doi: https://dx.doi.org/10.1590/s0100-204x2017000300004

BARBOSA JC, MALDONADO JÚNIOR W. Agroestat: Sistema para análises estatísticas de ensaios agronômicos [System for statistical analysis of agronomic trials]. Jaboticabal: Unesp. 2010.

BEWLEY, J.D.; BRADFORD, K.J.; HILHORST, K.H.W.M.; NONOGAKI, H. Seeds: Physiology of development germination and dormancy. New York: Springer, 392p., 2013.

CAGNA, C. P.; CALÁBRIA, Z. K. P.; GUEDES FILHO, O.; PACHECO, L. P., \& SILVA, T.J.A. Structural properties of soil in maize and forage grass intercropping under no-tillage in the Brazilian cerrado. Engenharia Agrícola, 39(4), 512-517. Epub August 12, 2019. Disponível em: https://dx.doi.org/10.1590/1809-4430eng.agric.v39n4p512-517/2019

CARVALHO, P.C.F.; PETERSON, C.A.; NUNES, P.A.A.; MARTINS, A. P.; SOUZA FILHO, W. et al. Animal production and soil characteristics from integrated croplivestock systems: toward sustainable intensification, Journal of Animal Science, v. 96, n. 8, p. 3513-3525, 2018. Disponível em: https://doi.org/10.1093/jas/sky085 
CAVARIANI, C.; NAKAGAWA, J.; VELINIL, E.D. Mistura de fertilizantes fosfatados com sementes de Brachiaria decumbens Stapf e Brachiaria brizantha (Hochst. Ex A. Rich) Stapf. Revista Brasileira de Sementes, v.16, n.2, p.163-167, 1994.

CODOGNOTO, L.; CONDE, T.T.; MALTONI, K.L.; FARIA, G.M.; REIS, A.R. Physiological response of marandu grass seeds exposed to NPK fertilizer. Ciência Rural, v. 49, n.6, 2019. Disponível em:< http://dx.doi.org/10.1590/01038478cr20180084> http://dx.doi.org/10.1590/0103-8478cr20180084

DAN, H.A; DAN, L.G.M; BARROSO, A.L.L; BRACCIN, A.L; PICCININ, G.G. Mistura de sementes de Brachiaria ruziziensis com uréia visando a implantação do sistema de integração lavoura-pecuária. Revista Caatinga, v.24, p.68-73, 2011

JOHANN, A. K.; BIGOLIN, G.; HARTER, L.S.H. Avaliação da qualidade fisiológica de sementes de linho (Linum usitatissimum L.) pelo teste de envelhecimento acelerado. Enciclopédia Biosfera, Centro Científico Conhecer- Goiânia, v.16 n.29; p. 323-338 2019. Disponível em DOI: 10.18677/EnciBio_2019A24

LIMA, E.V; TAVARES, J.C.S; AZEVEDO, V.R; LEITÃO-LIMA, P.S. Mistura de sementes de Brachiaria brizantha com fertilizante NPK. Ciência Rural, v. 40, n. 2, 441-444, $2010 . \quad$ Disponível em: <http://dx.doi.org/10.1590/S0103$84782010005000003>$

LIMA, E.V; TAVARES, J.C.S; AZEVEDO, V.R; LEITÃO-LIMA, P.S; PINHEIRO, et al. Germinação e vigor de sementes de Brachiaria brizantha cv. Marandú em função do tempo de mistura com ureia. Revista de Agricultura, v.89, n.1, p. 41 - 52, 2014.

LOPES, A. S.; GUiLHERME, L. R. G. A career perspective on soil management in the Cerrado Region of Brazil. Advances in Agronomy, v.137, p.1-72. 2016. Disponível em:<https://doi.org/10.1016/bs.agron.2015.12.004>

MATEUS, G. P.; BORGHI E.; MARQUES, R.R.; BOAS, R.V.; CRUCIOL, C.A.C.. Fontes e períodos de contato de fertilizantes e germinação de sementes de Brachiaria brizantha. Revista Brasileira de Ciência do Solo, v. 31, n 1, p. 177-183, 2007. Disponível em: < DOI:10.1590/s0100-06832007000100018>

MATEUS, G. P., C. A. C. CRUSCIOL, C. M. PARIZ, E. BORGHI, C. COSTA, J. M. et al.Sidedress Nitrogen Application Rates to Sorghum Intercropped with Tropical Perennial Grasses. Agronomy Journal. 108:433-447. 2016 doi:10.2134/agronj2015.0236

MORAES, M. T.; DEBIASI, H.; CARLESSO, R.; FRANCHINI, J. C.; SILVA, V. R. et al. Soil physical quality on tillage and cropping systems after two decades in the subtropical region of Brazil. Soil \& Tillage Research, v. 155, p. 351-362, 2016. Disponível em: <https://doi.org/10.1016/j.still.2015.07.015>

PANISON, F., SANGOI, L., DURLI, M.M.; LEOLATO, L.S., COELHO, A.E. et al., Timing and Splitting of Nitrogen Side-Dress Fertilization of Early Corn Hybrids for 
High Grain Yield. Revista Brasileira de Ciência do Solo, 43, e0170338. 2019. Epub March 28, 2019.https://dx.doi.org/10.1590/18069657rbcs20170338

PERES, A.; VAZQUEZ, G.H.; CARSOSO, R.D. Physiological potential of Brachiaria brizantha cv. Marandu seeds kept in contact with phosphatic fertilizers. Revista Brasileira de Sementes, v. 34, n. 3, 424-432, 2012. Disponível em: < http://dx.doi.org/10.1590/S0101-31222012000300009>

PHILP, J.N.M.; VANCE, W.; BELL, R.W.; CHHAY, T.; BOYD, D. et al. Forage options to sustainably intensify smallholder farming systems on tropical sandy soils. A review. Agronomy for Sustainable Development. v.39, n.30, 2019. Disponível em: https://doi.org/10.1007/s13593-019-0576-0

RIBEIRO, E.C.; REIS, R.; VILAR, C.; VILAR, F.C. Qualidade fisiológica de sementes de Urochloa brizantha submetidas a condicionamento com sais de cálcio. Pesquisa Agropecuária Tropical (Agricultural Research in the Tropics), v. 49, p. e55341, 9 jul. 2019. Disponível em: < https://www.revistas.ufg.br/pat/article/view/55341/33343>

SILVA, D.C.B.; OLIVEIRA, G.P.; RAGONHA, E.P. Potencial fisiológico de Convert hd364, em diferentes períodos de contato com adubo. Revista Thema. v.15, n.2. 2018. Disponível em:< http://dx.doi.org/10.15536/thema.15.2018.531-538.884> doi/10.15536/thema.15.2018.531-538.884

SPACKMAN, J. A., F. G. FERNANDEZ, J. A. COULTER, D. E. KAISER, AND G. PAIAO. 2019. Soil Texture and Precipitation Influence Optimal Time of Nitrogen Fertilization for Corn. Agronomy Journal. 111:2018-2030. doi:10.2134/agronj2018.09.0605

TAFFAREL, L. E.; OLIVEIRA, P.S.R. DE; PIANO, J.T.; COSTA, P.B.; MESQUITA, E.E. et al. Agronomic characteristics and nutritional value of oats and corn intercropped with brachiaria in a crop-livestock integration system. Acta Iguazu 2018 Vol.7 No.3 pp.1-16. 2018. Disponível em http://e-revista.unioeste.br/.../13374

TORRES, F. N.; RICHTER, R.; VOHLAND, M.A.; A Multisensoral approach for highresolution land cover and pasture degradation mapping in the humid tropics. A case study of the fragmented landscape of Rio de Janeiro, International Journal of Applied Earth Observation and Geoinformation, v.78, p. 189-201, 2019. doi 03032434, Disponível em: https://doi.org/10.1016/j.jag.2019.01.011. (http://www.sciencedirect.com/science/article/pii/S0303243418308882)

VALLE JÚNIOR, R.F.; SIQUEIRA, H. E.; VALERA, C. A.; OLIVEIRA, C. F; SANCHES, L. F. F. et al. Diagnosis of degraded pastures using an improved NDVIbased remote sensing approach: An application to the Environmental Protection Area of Uberaba River Basin (Minas Gerais, Brazil). Remote Sensing Applications: Society and Environment, v. 14, p. 20-33, 2019. Disponível em: https://doi.org/10.1016/j.rsase.2019.02.001 